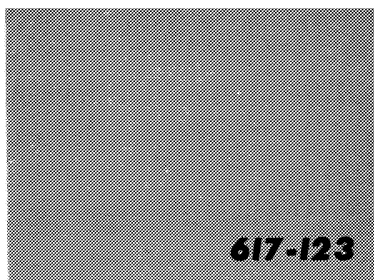 \\ Normalización del cemento
}

Notas y explicaciones respecto a la reelaboración de la norma alemana del cemento DIN 1164; redacción junio 1970

6. WISCHERS

Beton, n० 4, abril 1971. pdigs. $147-150$

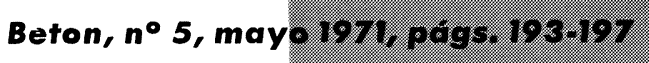

\section{VISION PANORAMICA}

La norma de cemento DIN 1164 tiene que cumplir diferentes misiones; es a la vez norma de material, ensayo, calidad y suministro. La DIN 1164 trata de cemento portland, cemento portland de escoria, de horno alto y puzolánico. Estos cementos se distinguen por su composición; no obstante, se juzgan de acuerdo con los mismos ensayos y deben satisfacer dentro de las diferentes clases a las mismas exigencias. Son también compatibles entre sí.

La mayor parte del cemento se convierte en hormigón. Por ello, al usuario del cemento le interesa, en primer lugar, su comportamiento bajo esta forma, especialmente la contribución a su desarrollo resistente. Como las propiedades del hormigón dependen de varias magnitudes de influencia, la influencia del cemento sólo puede comprobarse en una mezcla de hormigón "normal" constantemente idéntica, lo que dificultaría el estudio normativo de los cementos aun cuando no fuera más que por la cantidad y costes en relación con el árido normal idéntico en todas partes. Por eso, el comportamiento del cemento en el hormigón se examina y juzga indirectamente mediante el procedimiento de examen de analogía. El fraguado se estudia en la pasta de cemento, igual que la estabilidad de volumen y el calor de hidratación. Para el estudio de la resistencia se emplea un mortero normal. Las demás propiedades, como, por ejemplo, la resistencia a los sulfatos, se juzgan según la composición del cemento.

La innovación más importante en la norma de cemento DIN 1164, redacción junio 1970, es, sin duda alguna, la restricción adicional superior de la resistencia en cada una de las clases. Mediante la delimitación de las resistencias inferior y superior se logra una elevada regularidad de la resistencia de 28 días. Como en el examen y control no debe haber ningún valor individual fuera de los límites de resistencia, la resistencia media de todo cemento debe encontrarse aproximadamente en el centro del campo establecido para las distintas clases. En el caso de una desviación standard de $30 \mathrm{kp} / \mathrm{cm}^{2}$ (dispersión de ensayo y de calidad), el $90 \%$ de todos los valores de ensayo de un cemento se desviarán menos de $\pm 50 \mathrm{kp} / \mathrm{cm}^{2}$ de su valor medio. Esto representa una seguridad muy elevada y totalmente suficiente para el cumplimiento de una determinada resistencia en el hormigón, 
si en el cálculo de la mezcla se parte del valor medio del campo de resistencias de la clase de cemento empleado.

Esta nueva regulación sirve, no sólo para mejorar la regularidad y seguridad, sino que al simplificar se fomenta también la racionalización y unificación de la fabricación del hormigón.

\section{GENERALIDADES}

El cemento como producto no se puede emplear directamente, lo mismo que le ocurre por ejemplo, al vidrio, los ladrillos o el acero, sino que es el material de partida para fabricar el material de construcción propiamente dicho. Las formas de aplicación se extienden desde el mortero y hormigón, pasando por la estabilización y mejora del suelo e inyecciones, hasta llegar al campo de las pinturas; la elaboración del cemento como hormigón constituye seguramente más de las tres cuartas partes de su utilización.

La fabricación y elaboración del hormigón se transforma, constantemente, de una actividad originalmente artesana en una fabricación cada vez más industrializada. Causa de esto son la racionalización impuesta por el creciente aumento de los costes y el esfuerzo por la preparación de hormigones de mejor calidad. Mano a mano con el desarrollo van también la tecnología y las instalaciones necesarias para conseguirlo, es decir, los centros de preparación y elaboración. La fabricación del hormigón a escala industrial ha adquirido un gran desarrollo en los últimos decenios. En la actualidad, el $30 \%$ aproximadamente del cemento se consume en productos y elementos fabricados de hormigón; otro $30 \%$ aproximadamente en hormigón de central, y en numerosas obras medianas y grandes se emplean instalaciones móviles muy desarrolladas para la preparación del hormigón.

La creciente demanda de calidad, homogeneidad y economía del hormigón condicionan, no sólo instalaciones más caras de fabricación, sino, consecuentemente, mayores demandas de los materiales de partida, es decir, del cemento y de los áridos.

La fabricación industrial del material de construcción hormigón en masa exige un material de partida, un cemento, homogéneo, siempre regular en sus propiedades. En relación con esto y para la mayor parte de los casos, cada vez tiene menos fuerza esta o aquella peculiaridad de un cemento determinado.

Junto a esto, la multiplicidad de los campos de aplicación provoca asimismo una creciente búsqueda de propiedades adicionales especiales. Las propiedades especiales pueden ser útiles en circunstancias muy concretas, como por ejemplo en la fabricación del hormigón centrifugado, o pueden resultar necesarias para elementos de construcción, a los que se plantean exigencias respecto de la capacidad de resistencia química o del color.

Todo esto quiso tenerse en cuenta al reelaborarse la norma del cemento DIN 1164, que había sido adoptada desde hace más de 10 años y que concluyó a mediados del pasado año, hasta el punto que permite el estado actual de la técnica del cemento.

\section{CEMENTO}

\section{Producto de la industria de rocas $\mathrm{y}$ arcillas}

El cemento es un conglomerante hidráulico que, en forma de pasta (mezcla de cemento 
y agua), une los granos del árido. Este conglomerante necesita solamente agua, se endurece a continuación de manera autónoma tanto al aire como bajo el agua y permanece resistente al agua así como consistente bajo todas las condiciones habituales del mundo circundante. Ningún otro conglomerante orgánico o inorgánico presenta juntas estas peculiaridades.

En el año 1970 se fabricaron en la República Federal de Alemania unos 35 millones de toneladas de cemento. Tanto al volumen como a la multiplicidad en sus aplicaciones contribuye, seguramente, el bajo precio, que en fábrica, a pesar de todos los aumentos de costo de los últimos años, sigue estando por debajo de $0,05 \mathrm{DM} / \mathrm{kg}$. Esto ha sido posible gracias al desarrollo reciente de la técnica de los procedimientos de fabricación de cemento que, aunque ha conducido a elevadas inversiones, ha reducido, sin embargo, el trabajo empleado a menos de 1 hora por tonelada de cemento. Las grandes unidades mecánicas - horno de cocción rotatorio con una producción de más de 3.000 t/día y molinos de cemento con más de $100 \mathrm{t} / \mathrm{h}$ - han contribuido de manera esencial, juntamente con la refinada técnica de medida y regulación, a la homogeneización de las cualidades del cemento.

Sin embargo, hay que observar que en el caso que nos ocupa se trata de un producto a granel de la industria de las rocas y tierras, cuya materia prima son productos poco uniformes, que se preparan, calcinan e, incluso, se funden. Hay que tolerar ciertas peculiaridades de los yacimientos porque no pueden modificarse con un gasto económicamente rentable. Es cierto que, dentro de ciertos límites, es posible completar los elementos que faltan; sin embargo, es difícil sustraer antes de la elaboración los materiales que se presentan con excesiva proporción en las materias primas.

Los cementos pueden controlarse perfectamente en propiedades esenciales, como por ejemplo, en la resistencia a la compresión a 28 días; pero otras propiedades, como por ejemplo las resistencias a edades más tempranas, están delimitadas por los yacimientos de materias primas y sólo pueden modificarse dentro de ciertos límites. En el caso de propiedades menos importantes, como por ejemplo la finura de molienda, hay variaciones que desde el punto de vista de la técnica de la construcción apenas pueden percibirse, y son hasta inevitables, pero la finura es una magnitud importante de regulación en relación con una resistencia homogénea a 28 días.

\section{METODOS DE ENSAYO}

\section{Base de la normalización}

Ya en la primera norma del cemento que data del año 1877 se contenían ensayos y exigencias para las tres propiedades características y más importantes del cemento (fraguado lento, desarrollo de resistencias y estabilidad después del endurecimiento); por su importancia se mantienen todavía hoy como el fondo esencial de la más reciente norma del cemento.

En Alemania tampoco se ha modificado la naturaleza de la norma del cemento a través de los muchos años; es una norma, entre otras cosas, de material, de ensayo, de calidad y de suministro conjuntamente (1).

(1) Fundamentalmente hay que distinguir entre normas de medidas, normas de material, normas de calidad, normas de ensayo, normas de suministro, etc. Muchas normas incluyen, no obstante, varias de estos tipos. 
Las normas de calidad y suministro presuponen procedimientos de revisión y criterios a los que se refieren las demandas de calidad y que sirvan para comprobar la obligación de suministro.

Las esperanzas del consumidor se centran inmediatamente en el material de construcción fabricado con cemento, es decir, en el hormigón y sólo de manera mediata al cemento, del que, sin embargo, se espera una contribución decisiva en favor de las propiedades deseadas del hormigón. Como las propiedades del hormigón dependen de muchos factores de influencia, sólo con las propiedades del cemento no se garantizan sin más determinadas propiedades del hormigón; para ello hay que cumplir otras condiciones tecnológicas en este material. Por la misma razón, tampoco se pueden sacar conclusiones de las propiedades del hormigón refiriéndose solamente a las propiedades del cemento, tal como se suele suponer erróneamente con frecuencia en la práctica.

Por otra parte existe un interés justificado del consumidor en cómo contribuye el cemento a las propiedades del hormigón, especialmente a su resistencia mecánica. Los procedimientos de ensayo en relación con el cemento y las exigencias de calidad referidas al mismo deben ser, por lo tanto, una medida de las propiedades técnicas en su aplicación en la industria del hormigón.

En relación con una norma de calidad y suministro bastarían métodos de ensayo que afecten tan sólo a las propiedades del cemento en la técnica de la construcción. No obstante, la norma del cemento es también una norma del material, que da un marco a la producción y en la que se pueden alcanzar las propiedades técnicas deseadas. No basta, por consiguiente, la mera revisión del cemento a posteriori, una vez fabricado ya que, por ejemplo, en el punto de origen de la producción deben aplicarse, a partir de la composición química del material crudo o de su finura, múltiples mediciones, controles y regulaciones.

En general, todo procedimiento de análisis debe responder a tres condiciones básicas:

En primer lugar, debe anunciar cúal es el objetivo de la prueba; esto es evidente en sí, pero no obstante se cuida poco con frecuencia en el desarrollo del procedímieto de ensayo. Los procedimientos de análisis que al enjuiciar las propiedades esenciales permiten una matización gradual, son en general más significativos. En el caso de condiciones marginales, que son menos esenciales, cuya existencia es solamente una presuposición de la utilidad, las llamadas pruebas de sí-no, pueden ser hasta más útiles.

Los procedimientos de revisión deben presentar además, en todo caso, suficiente reproducibilidad; es decir, si con el mismo material se realiza la misma prueba, en cualquier lugar que se efectúe el ensayo deben obtenerse los mismos resultados con la menor desviación posible. Naturalmente con una dispersión más reducida aumenta la exactitud del enjuiciamiento del producto. No obstante, no es útil ni razonable dar primacía a la reproducibilidad de un procedimiento de ensayo a costa de su misión propiamente dicha, ya que un procedimiento de enșyo muy significativo, pero de una reproducibilidad más bien moderada, puede ser mucho más útil que el caso contrario.

En tercer lugar, un procedimiento de ensayo debe ser lo más sencillo posible y de un gasto limitado. Esto último puede aplicarse, sobre todo, a los productos relativamente baratos como, por ejemplo, el cemento. Con frecuencia una reproducibilidad que excede la medida mínima requerida sólo puede lograrse con costes esencialmente más elevados.

Con vistas a la especial significación de un procedimiento de revisión para enjuiciar el cemento desde el punto de vista de la técnica de la construcción, es natural la idea de que se examine el cemento a través del hormigón. Como las propiedades del hormigón no de- 
penden sólo del cemento, sino también en la misma o mayor medida de otras magnitudes de influencia, como, por ejemplo, la relación agua/cemento (A/C), todos estos otros factores de influencia deberían nivelarse, lo que conduciría a una mezcla de hormigón normal que habría que establecer para la revisión del cemento. Por lo que conoce el autor, este camino - sin embargo, por otras razones- hasta ahora solamente se ha emprendido en Gran Bretaña en 1958, con motivo de una revisión de la resistencia del cemento [1]. En este caso no se ha prescrito ningún árido normalizado para el hormigón, sino que se puede emplear un árido natural disponible en cualquier parte, que corresponda a determinadas propiedades. Pero no se puede desviar la atención del hecho de que los diferente áridos pueden dar lugar, en este caso, a diferencias sistemáticas en la resistencia.

Resulta problemático si una mezcla de hormigón normalizado es más instructiva en cuanto a la contribución del cemento a la resistencia en el hormigón que una mezcla de mortero normal, compuesta, razonablemente, como la prescriben todas las normas de cemento, especialmente porque también la mezcla de hormigón normal prevé solamente una relación A/C determinada. La reproducibilidad no podría ser en ningún caso mejor, sino más bien peor. En todo caso una revisión de este tipo sería más costosa, por una parte, a causa de las grandes cantidades de árido normal, y, por otra, por exigir probetas de ensayo, aparatos, recipientes de conservación y máquinas de ensayo mayores. Además, la revisión de otras propiedades técnicas de construcción, como el fraguado, la estabilidad de volumen o la resistencia a los sulfatos, exigiría un gasto extraordinariamente elevado con la mezcla de hormigón normal.

Por tanto, en la revisión y normalización del cemento, desde hace unos 100 años, se han examinado y enjuiciado las propiedades técnicas con vistas a la construcción no inmediatamente, sino a través de los llamados procedimientos de revisión por analogía, que son más o menos instructivos con vistas al comportamiento del cemento en el hormigón. En el caso de algunos métodos de ensayo las ideas de los especialistas divergen entre sí, sin que se hayan llegado hasta el presente a encontrar procedimientos sencillos y más aleccionadores; sin embargo, son suficientes y satisfacen a las demás exigencias de todo procedimiento de ensayo, es decir, tienen una reproducibilidad suficiente, son las más de las veces sencillos y tampoco requieren un gasto exagerado.

\section{CEMENTO}

\section{Componentes principales y tipos de cementos}

El cemento molido finamente consta, esencialmente, de determinadas combinaciones de óxido de calcio, dióxido de silicio, óxido de aluminio y óxido de hierro que, en general, se han originado artificialmente por sinterización o fusión, o que, en sitios aislados, se encuentran como material puzolánico (trass) en la naturaleza. La norma de cemento alemana DIN 1164, edición junio 1970, menciona como elementos principales, el clínker de cemento portland, las arenas metalúrgicas (escoria de horno alto granulada) y trass, y como elementos secundarios, entre otros, la adición de sulfato de calcio para regular el fraguado.

\subsection{Clínker de cemento portland}

El clínker de cemento portland se origina por la cocción, hasta sinterización, de una mez- 
cla finamente molida de piedra caliza y arcilla, que en la naturaleza aparece como marga más o menos caliza. Portadores del endurecimiento son, fundamentalmente, las combinaciones de cal con el ácido silícico que se crean en la cocción. los silicatos de calcio. El crudo debe poseer, no obstante, una pequeña proporción de óxidos de aluminio y de hierro, porque así los silicatos cálcicos se originan con un gasto técnico y económico rentable en la cocción. El clínker de cemento portland finamente molido es autoendurecible.

En el caso del cemento, como producto fabricado en grandes cantidades, no se puede opinar que sea totalmente homogéneo, es decir, que todo gránulo presente exactamente la misma composición química o que siempre se calcine de la misma manera. Son inevitables insignificantes diferencias. No obstante para que la proporción de materiales incocidos procedentes de la fábrica sea tan pequeña que no tenga efectos perjudiciales, la nueva norma delimita la pérdida al fuego, el contenido en dióxido de carbono y el residuo insoluble del cemento preparado. Esto es extensible también a las posibles impurezas incorporadas en el yeso natural empleado para la regulación del fraguado. El contenido de elementos incocidos permitidos excluye con seguridad toda influencia perniciosa. (Como única norma de cemento, la DIN 1164 dispone, de acuerdo con esto, un límite para el contenido de dióxido de carbono. Los carbonatos, que no pueden descubrirse como tales en la disgregación con ácido clorhídrico, son efectivamente delimitados).

\subsection{Arenas metalúrgicas}

A la escoria de horno alto granulada se le denomina arena metalúrgica; se forma por el enfriamiento rápido de la escoria licuada procedente de la obtención del arrabio. Las escorias ácidas son, por lo general, menos adecuadas. Por ello, la norma alemana dispone que la relación en peso de cal + magnesia + óxido de aluminio a ácído silícico sea $\geqslant$ que 1. Esta fórmula excluye las arenas metalúrgicas que en su mezcla con el clínker de cemento portland (por ejemplo en forma de cemento portland férrico o de alto horno) no contribuyen o contribuyen poco al endurecimiento. Por otra parte, no todas las arenas metalúrgicas que corresponden con la fórmula son adecuadas. Por esta razón, las normas de cemento extranjeras no utilizan este criterio de enjuiciamiento. La arena metalúrgica, hidráulica en estado latente, finamente molturada, requiere un excitante para desarrollar suficientemente su propia hidraulicidad. En Alemania, y para obtener cementos normales, sólo se permite como excitante el clínker de cemento portland. Son posibles otros excitantes, como, por ejemplo, la cal y el sulfato, pero estos proporcionan en conjunto menos ventajas.

\subsection{Trass}

El trass es un material natural con propiedades puzolánicas, o sea, por su reacción con la cal proporciona compuestos hidráulicos. El cemento puzolánico normalizado es una mezcla finamente molida de clínker de cemento portland y puzolana, en la que la cal desprendida durante la hidratación del clínker de cemento portland crea combinaciones hidráulicas con la puzolana. El trass o puzolana debe corresponder a las exigencias de la norma DIN 51043. Su actividad puzolánica se comprueba con un mortero de trass y cal, que por conservación bajo agua debe alcanzar determinadas resistencias mínimas.

En Italia no se estudia el material puzolánico aisladamente, sino que el ensayo de idoneidad se hace sobre el cemento puzolánico ya preparado, el cual debe presentar, en primer lugar, una resistencia suficiente; y en segundo lugar, como comprobación de la reacción 
de la puzolana con la cal, el contenido de ésta en una suspensión de agua y cemento debe ser inferior a la saturación.

\subsection{Tipos de cemento}

Los diferentes tipos de cemento normalizados se distinguen, desde el punto de vista de su composición, por las proporciones de los tres elementos constitutivos. En la nueva norma alemana se han aceptado sólo mezclas de clínker de cemento portland y uno de los otros elementos principales que no endurecen de manera autónoma (arena metalúrgica, o trass, respectivamente). Otros cementos como el cemento de trass alto horno y el cemento de esquistos bituminosos que, eventualmente, se producen sólo en un estado federal, se pueden emplear si cumplen o se autorizan, por la vigilancia de la construcción, para los elementos a base de hormigón y hormigón armado para estructuras.

T A B L A 1

Composición de los cementos normales según DIN 1164 (junio 1970)

Suma de clínker de cemento portland y arena metalúrgica o trass igual a $100 \%$.

\begin{tabular}{|c|c|c|c|}
\hline & $\begin{array}{l}\text { Clinker de cemento } \\
\text { portland }\end{array}$ & Arena metalúrgica & Trass \\
\hline & $\%$ en peso & $\%$ en peso & $\%$ en peso \\
\hline Cemento portland & 100 & - & - \\
\hline Cemento portland férrico & $65 \ldots 99$ & $1 \ldots 35$ & - \\
\hline Cemento de horno alto & $15 \ldots 64$ & $36 \ldots 85$ & - \\
\hline Cemento puzolánico & $60 \ldots 80$ & - & $20 \ldots 40$ \\
\hline
\end{tabular}

En cuanto a los principales elementos, los cuatro tipos de cemento normales presentan la composición establecida en la tabla 1.

Las propiedades características de la arena metalúrgica se manifiestan con más intensidad en las propiedades del cemento cuanto mayor sea su proporción; prácticamente existe una transición continua desde el cemento portland, pasando por el cemento portland férrico (con reducido contenido de arena metalúrgica), al cemento de horno alto rico en arena metalúrgica con una proporción de 70 hasta $85 \%$. El contenido-límite de arena metalúrgica, establecido en la nueva norma de cemento alemana entre el cemento portland férrico y el de horno alto, del $35 \%$ está condicionado menos por las propiedades técnicas de construcción que por la recomendación $\mathrm{n}^{\circ} 771$ de la ISO. Tampoco existe fundamento técnico relacionado con la construcción para el contenido mínimo de arena metalúrgica, fijado en algunos países en el $20 \%$. Frente a esto, los cementos de horno alto con gran contenido de arena metalúrgica, especialmente los que tienen una gran proporción (mayor del $70 \%$ ), tienen claramente especiales propiedades térmicas en la construcción, por ejemplo una elevada resistencia a los sulfatos y, en general, un bajo calor de hidratación. 
El cemento puzolánico debe presentar un contenido mínimo de puzolana del $20 \%$ para que las propiedades especiales del trass, por ejemplo su capacidad de retención de agua, se manifiesten de manera adecuada en el hormigón fresco.

En la fabricación del cemento portland de escoria de horno alto, y puzolánico, los clínkeres de cemento portland y la escoria siderúrgica o la puzolana deben molturarse conjuntamente en la fábrica. Desde el punto de vista técnico es posible también moler estos materiales por separado y, a continuación, mezclarlos homogéneamente. En la norma, no obstante, se ha aceptado solamente la molturación conjunta, porque de este modo se garantiza una distribución homogénea de los materiales a la salida del molino. En otros casos esta condición previa sólo sería posible con un procedimiento especial, que hasta la fecha no se ha experimentado en la industria del cemento. Es misión de una norma recoger la forma más frecuentemente empleada y segura, y dejar que los procedimientos especiales sigan otro camino a través de un permiso especial.

\section{FRAGUADO}

El fraguado del hormigón depende de muchos factores de influencia. Es cierto que el cemento realiza un "atiesamiento" inicial, que pasa a un endurecimiento creciente ; sin embargo, pueden manifestarse otras influencias, como, por ejemplo, la consistencia, la temperatura o la relación $\mathrm{A} / \mathrm{C}$ con una intensidad fundamentalmente mayor en el tiempo que el comportamiento de los diversos cementos con respecto al fraguado.

La práctica de la construcción plantea la exigencia de que, tras el amasado, el hormigón siga siendo manejable durante un tiempo suficientemente prolongado. Las opiniones son divergentes en cuanto a qué período de tiempo es adecuado para ello. Es díficil un enjuiciamiento uniforme en el caso del hormigón, porque, hasta el momento, no hay un procedimiento universalmente aceptado para determinar su fraguado. Por medio de la medida de consistencia en determinados intervalos de tiempo se puede calcular el fraguado del hormigón; sin embargo, no hay reglas para la realización y evaluación. De todos modos la norma americana ASTM C 403-70 [2] presenta un procedimiento, con el que se mide el fraguado por la penetración de una varilla en el mortero fino de hormigón.

El fraguado del cemento se realiza prácticamente en todo el mundo con el aparato de aguja según Vicat (ver DIN 1164 pág. 5), en el que se mide la resistencia de una pasta de cemento, amasada sólo con agua, contra la penetración de una aguja cilíndrica de $1 \mathrm{~mm}^{2}$ de sección. (Las normas americanas de ensayo mencionan, junto al aparato de Vicat, el aparato de Gillmore, que trabaja de acuerdo con un principio semejante).

La pasta de cemento recién amasada debe tener una cierta consistencia en el ensayo según Vicat, consistencia que se denomina normal y que se puede determinar con el mismo aparato, sustituyéndose la aguja de Vicat por un cilindro calibrado. De acuerdo con la finura de molturación del cemento, su composición y la intensidad en el amasado de la pasta, el cemento requiere una cantidad de agua distinta (entre 23 y. $30 \%$ ) para la consistencia normal.

Las definiciones convencionales de este método de ensayo se representan esquemáticamente en la figura 1. El punto de partida es una pasta de cemento de consistencia normal.

Al prolongarse el tiempo aumenta asimismo la resistencia a la deformación, primeramente poco, y después con más intensidad, hasta que el fraguado evoluciona hacia el endureci- 
miento. El comienzo del fraguado se define cuando la aguja de Vicat, con un peso de $300 \mathrm{~g}$, no atraviesa ya completamente una capa de pasta de $40 \mathrm{~mm}$ de espesor y se detiene a los 3 ó $5 \mathrm{~mm}$ del fondo del molde. La resistencia al final del fraguado debe ser tan grande que la aguja penetre a lo sumo $1 \mathrm{~mm}$ en la pasta solidificada. Mediante pruebas repetidas con la pasta de cemento que empieza a ponerse rígida, se determina el tiempo después del amasado hasta el comienzo del fraguado y hasta el final del mismo.

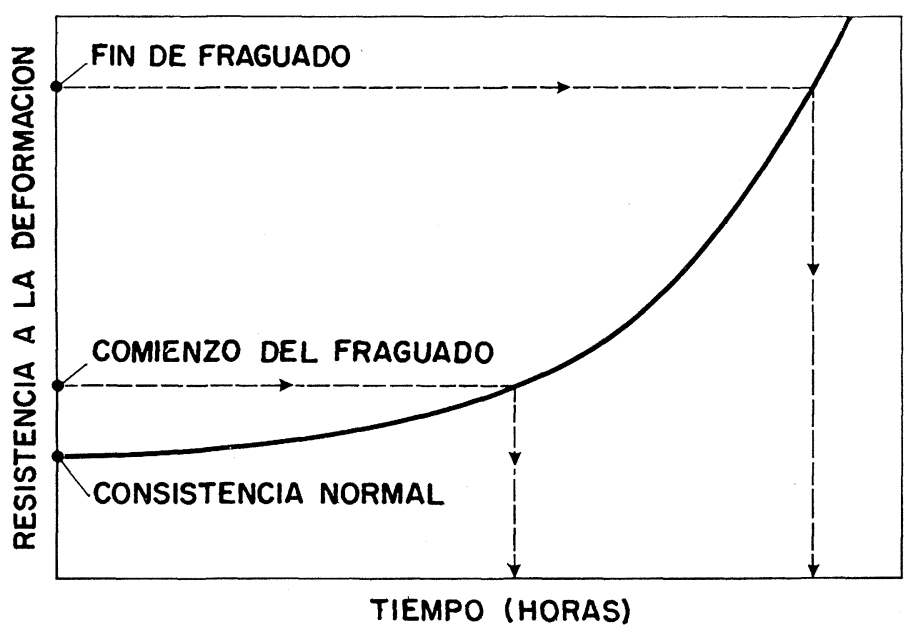

Fig. 1.-Representación esquemática del fraguado de la pasta de cemento (análisis del principio de fraguado y del final del mismo en un cemento).

El procedimiento de ensayo con el aparato de la aguja es sencillo y no requiere un gran gasto. La reproducibilidad no es, sin embargo, muy buena. Así, en un ensayo comparativo la desviación standard para el comienzo del fraguado se elevó a unos 30 minutos, lo que corresponde a un coeficiente de variación de más del $16 \%$. La DIN 1164, pág. 5, redacción de junio 1970, prevé por ello que la pasta de cemento no se mezcle a mano, sino durante 3 minutos en la amasadora de mortero, para mejorar la reproducibilidad.

No se ha estudiado, o al menos no se ha publicado, si los resultados obtenidos con el aparato de Vicat se pueden trasladar directamente al comportamiento del cemento en el hormigón, es decir, si, por ejemplo, un comienzo de fraguado de doble duración según Vicat da como resultado un tiempo de elaboración del hormigón también doble, porque, probablemente, no hay procedimientos adecuados para estudiar el fraguado del hormigón. Por eso, tampoco se puede discurrir inversamente: deducir de los requisitos de la práctica del hormigón los valores límite de acuerdo con el procedimiento de Vicat. De todos modos se puede enjuiciar si los resultados obtenidos hasta ahora corresponden a las necesidades prácticas. Como prescribe hasta la fecha la DIN 1164, texto de junio 1970, el comienzo del fraguado no debe tener lugar antes de 1 hora, y el final del mismo debe sobrevenir dentro de las 12 horas.

En general, en la técnica constructiva solamente tiene importancia el principio del fraguado. Determinar de que el principio de fraguado no debe acaecer antes de 1 hora, basta por lo general en la práctica. En relación con productos de hormigón con vistas a una rápida resistencia mínima reciente puede desearse un comienzo de fraguado, que va sólo un 
poco más allá de 1 hora. Inversamente, con vistas a un endurecimiento prematuro en vías de circulación puede ser útil un principio de fraguado, que tiene lugar más tarde.

En este contexto es interesante una comparación de 43 normas internacionales de cemento [3]. Cinco países permiten un principio de fraguado después de 30 minutos, casi un tercio de países después de 45 minutos y aproximadamente la mitad de todos ellos después de 1 hora. En un país se prescribe un principio de fraguado mínimo de 3 horas.

Aproximadamente un cuarto de países renuncian a una delimitación temporal del final de fraguado, lo que, según la opinión del autor, no debe tener consecuencias desventajosas, pues el cemento debe satisfacer, pasado un cierto tiempo, las exigencias de resistencia. La mitad de todos los países prescribe, sin embargo, un final de fraguado dentro de las 10 ó 12 horas (Alemania se encuentra entre ellos). Con vistas a la jornada de trabajo de 8 horas no es práctica la revisión del final de fraguado, que no tiene ninguna importancia desde el punto de vista de la técnica de la construcción en las circunstancias normales, de forma que en una reelaboración posterior habría que revisar esta deierminación.

T A B L A 2

Determinación del principio y final del fraguado en diferentes países según [3]

\begin{tabular}{|c|c|c|c|c|c|}
\hline \multicolumn{4}{|c|}{ Comienzo de fraguado } & \multicolumn{2}{|c|}{ Final de fraguado } \\
\hline \multicolumn{3}{|c|}{ Tiempo (minutos) } & \multirow{3}{*}{$\begin{array}{c}\text { n.o de países } \\
\begin{array}{c}5 \\
1\end{array}\end{array}$} & Tiempo (horas) & \multirow{2}{*}{$\frac{\text { n.o de países }}{2}$} \\
\hline no & antes $c$ & de 30 & & no antes de 3 & \\
\hline & $"$ & $" \quad 40$ & & $\begin{array}{llll} & \end{array} \quad, \quad, \quad 4$ & 1 \\
\hline$"$ & $"$ & $" \quad 45$ & 14 & no después de 6 & 1 \\
\hline$"$ & $"$ & $" \quad 60$ & 20 & no antes de 7 & 1 \\
\hline$"$ & $"$ & $" 120$ & 1 & no después de 8 & 3 \\
\hline$"$ & $"$ & $" 150$ & 1 & $" 10$ & 14 \\
\hline & $"$ & $" 180$ & 1 & $" 12$ & 8 \\
\hline & & & & $" 15$ & 2 \\
\hline
\end{tabular}

En conjunto, las especificaciones respecto al fraguado en la norma de cemento alemana de nueva redacción se limitan a la costumbre más extendida en el campo internacional.

\section{ENDURECIMIENTO DE LOS CEMENTOS}

\section{Tipos de resistencia}

La pasta de cemento solidificada adquiere una resistencia cada vez más elevada y se convierte de este modo en "piedra de cemento". La resistencia del hormigón normal viene determinada en gran medida por la relación A/C y la contribución del cemento. Tanto el método de ensayo en relación con la resistencia del cemento como la clasificación de los mismos en tipos resistentes basados en los resultados del ensayo, deben caracterizar la contribución de resistencia del cemento en el hormigón y esto de forma segura. 


\subsection{Método de ensayo de la resistencia del cemento}

Si se prescinde de la medida de la resistencia del cemento ya mencionada, permitida alternativamete en Inglaterra con la de un hormigón normal, en el mundo entero, por lo que conoce el autor, se analiza la resistencia del cemento en los llamados morteros normales. Las determinaciones en forma de mortero son, sin embargo, muy diferentes de unos países a otros. Las distintas determinaciones se deben fundamentalmente a la distinta respuesta de estas dos cuestiones:

1) Si ha de establecerse un mismo contenido de agua para todos los cementos o regularse una misma consistencia del mortero para todos los cementos;

2) si ha de establecerse la consistencia tan blanda que no sea necesario definir ninguna concentración, o si ha de establecerse exactamente la concentración en un mortero rígido.

Como la mayor parte de los demás países, la nueva norma alemana del cemento prevé un contenido constante de agua para el mortero normal. Esta decisión tiene como base la consideración de que las exigencias de agua del hormigón normal para una determinada consistencia viene definida, en primer lugar, por los áridos; por ello, puede despreciarse la influencia del cemento en la consistencia. Esto es cierto, seguramente, en el caso de hormigones con contenido de cemento reducido y medio, así como en el caso de cementos de finura reducida o media. Los hormigones muy ricos en cemento (más de $350 \mathrm{~kg} / \mathrm{m}^{3}$ ) de muy elevada finura (más de $5.000 \mathrm{~cm}^{2} / \mathrm{g}$ ) muestran claramente unas exigencias de agua mayores para una determinada consistencia. Las elevadas resistencias encontradas en el ensayo de cementos de elevada finura con un contenido constante de agua no dan en consecuencia frecuentemente una elevada contribución de resistencias en el hormigón, en las condiciones prácticas de construcción, por exigir mayores proporciones de agua. Como los cementos de una finura muy elevada sólo constituyen una pequeña parte de la producción, está justificado elegir, mediante un mortero de ensayo con contenido constante de agua, el juicio aplicable a los valores predominantes en los cementos.

Según la norma de ensayo ASTM C 109-70 no se establece como constante el contenido de agua, sino que se elige tan grande que el mortero de ensayo debe presentar la consistencia prescrita. Los cementos muy finos se ensayan, de acuerdo con esta norma, con una relación A/C más elevada por exigir más agua, en la mayor parte de los casos, que los cementos toscos y de finura media; por esto, proporcionan una resistencia normal relativamente menor que en el estudio con contenido constante de agua.

En el ensayo con un contenido constante de agua, éste es distinto en las normas del cemento de diferentes países. Expresado como relación A/C, las especificaciones se establecen en el intervalo comprendido etre 0,30 y 0,65. Como se trata siempre de morteros muy ricos en cemento, la consistencia de aquél viene determinada por la relación A/C. Así, con una relación $\mathrm{A} / \mathrm{C}$ de 0,30 se origina una pasta de cemento pastosa, pero con una relación 0,65 se forma una pasta muy fluida. En este caso, hay que tener en cuenta que ya las pastas con relaciones $\mathrm{A} / \mathrm{C}$ superiores a 0,40 , según el cemento, se inclinan más o menos a desprender agua (exudación).

La consistencia y el desprendimiento de agua dependen también de la arena normal empleada en el mortero de ensayo y, más concretamente, de la relación de mezcla cemento/arena normal y de la composición granulométrica de dicha arena normal. La relación de mezcla cemento/arena está fijada en todas las normas; en 38 de los 44 países, esta 
relación es de 1:3 [4]. En Japón está vigente la relación de mezcla 1:2; en los Estados Unidos y en los demás estados americanos se emplea una relación de 1:2,75. La nueva norma alemana del cemento - al igual que las ediciones anteriores- emplea una proporción de mezcla de 1:3, lo mismo que en la recomendación ISO n. ${ }^{\circ} 772$. Esta recomendación ISO reproduce el método de ensayo, elaborado en los años 50 por los Institutos de Cemento del occidente europeo agrupados en el Cembureau y aceptado también por RILEM.

La composición granulométrica de las arenas normales es distinta en las diferentes normas, al menos en parte, tanto en lo que se refiere al tamaño máximo, como al desarrollo de la curva granulométrica y a las desviaciones permitidas. La nueva arena normal alemana presenta un diagrama granulométrico continuo, con desviaciones tolerables estrictamente delimitadas y un tamaño máximo de $2 \mathrm{~mm}$. La arena de la norma alemana existente hasta ahora (edición 1942) se componía de dos grupos de granulometría estrictamente definidos con un tamaño máximo de $1,25 \mathrm{~mm}$ y el correspondiente límite en los finos. La nueva arena normal con una distribución continua tiene la ventaja de que su capacidad de retención de agua es mayor que la de la arena normal existente hasta la fecha, es decir, actúa en contra de la posible segregación de agua del mortero de ensayo.

La norma alemana de cemento anterior a 1942 prescribía una relación $\mathrm{A} / \mathrm{C}$ de 0,32 , que proporcionaba un mortero normal con consistencia de tierra húmeda. Este mortero, muy seco, debía someterse a una consolidación intensa (compactación con un aparato a martillo). En la norma de cemento existente hasta hoy día (edición 1942), la relación A/C era de 0,60. En este caso se originaba un mortero blando, que se podía compactar fácilmente con un pisón a mano y donde el tipo e intensidad de la compactación no tenía prácticamente ninguna influencia en el resultado. Por otra parte, por su elevada relación A/C y por la poco favorable composición granulométrica de la arena este mortero era propenso a la exudación de agua, tanto más cuanto más baja era la capacidad de retención de ésta por el cemento. Tras la pérdida de agua del mortero de ensayo compactado de esta forma, la verdadera relación $\mathrm{A} / \mathrm{C}$ no se encontraba en la relación establecida en el amasado, es decir, en 0,60 , sino por debajo, y — según el cemento- llegaba hasta 0,50 o incluso menos en algunos casos particulares.

El mortero normal de la nueva norma alemana del cemento no tiene esta desventaja del desprendimiento de agua en distinta forma, porque su relación $\mathrm{A} / \mathrm{C}$ se ha disminuido a 0,50 y porque la arena normal por su distribución granulométrica continua tiene una mejor capacidad de retención de agua. Por otra parte, este mortero no es ya blando, sino plástico, y necesita una compactación definida. La recomendación ISO propone para la compactación una mesa de choque; en la nueva norma alemana se ha prescrito para esto una mesa vibratoria que proporciona los mismos resultados, pero que es más práctica para su manejo. La experiencia anterior ha mostrado que, en comparación con los resultados anteriores, los morteros de ensayo actualmente más consistentes recogen algo de aire en la mezcla, y la eliminación total de los poros más finos de aire es difícil, incluso con una intensa compactación. Hay que atribuir a esto una parte de las dispersiones del ensayo.

Para juzgar la expresividad del procedimiento de ensayo se examinaron 29 cementos, representativos de todos los tipos fabricados en Alemania y de todas las clases de resistencia, según la nueva DIN 1164 (1970), la antigua DIN 1164 (1942) y la ASTM C 109. Además, con todos los cementos se fabricó un hormigón con un contenido de cemento de 300 $\mathrm{kg} / \mathrm{m}^{3}$ y una relación $\mathrm{A} / \mathrm{C}$ de 0,60 como composición más representativa de la práctica. Para la evaluación se estudió, en primer lugar, la resistencia a la compresión a los 28 días, 
tanto del cemento como del hormigón. Esta evaluación no hace justicia al procedimiento de ASTM C 109, porque en el estudio del cemento se eligió la misma consistencia del mortero de ensayo, y en el ensayo del hormigón, por el contrario, se empleó siempre la misma composición sin tener en cuenta la consistencia del hormigón. Las diferencias en la consistencia del hormigón no fueron, sin embargo, muy grandes, de forma que una corrección no hubiera influido apreciablemente sobre el resultado.

La figura 2 contiene los resultados; en la ordenada se registra la resistencia a la compresión del hormigón y en la abscisa la resistencia a la compresión del cemento, según los tres procedimientos de ensayo.
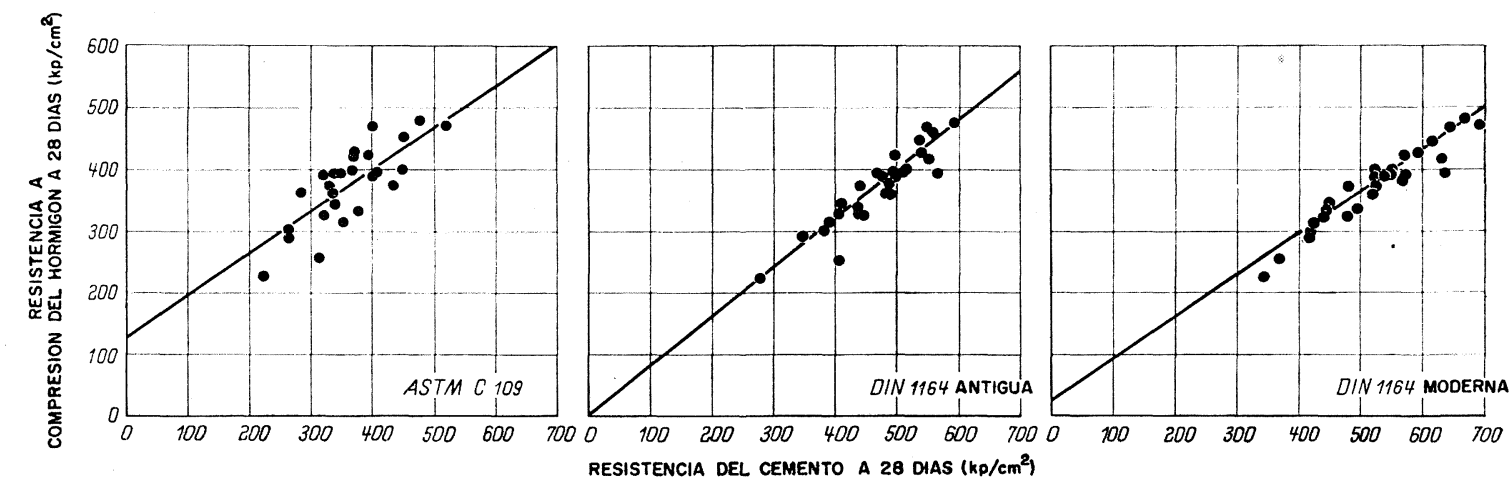

Fig̀. 2.-Relación entre la resistencia del hormigón a la compresión a 28 días y la resistencia a la compresión del cemento a 28 días, según ASTM C 109, DIN 1164 antiguo (1942) y DIN 1164 nuevo (1970) (corresponde al procedimiento ISO).

Las regresiones lineales entre la resistencia del hormigón y la resistencia eventual del cemento proporcionaron las rectas inscritas en la figura 2. La expresividad de un procedimiento de ensayo del cemento, en cuanto a su contribución a la resistencia que proporcionan cada uno de las cementos en el hormigón, es tanto mayor cuanto más cerca de las rectas de regresión se encuentran los puntos de medida.

La medida del ensayo representa, a este respecto, un enjuiciamiento numérico. Para los tres procedimientos de ensayo del cemento se calculó: para ASTM C 109, en $56 \%$; para DIN 1164 antiguo (1942), en $85 \%$, y para DIN 1164 nuevo (1970), en $91 \%$. En todo proceso técnico, las medidas por encima del $80 \%$ significan ya una elevada garantía de seguridad y una base para los procesos regulares. El procedimiento de ensayo correspondiente a las recomendaciones ISO y, por tanto, a la nueva norma alemana del cemento DIN 1164, posee una gran expresividad en cuanto a la contribución de resistencia del cemento en el hormigón. También el procedimiento de ensayo según DIN 1164 antiguo (1942), adoptado hasta ahora, correspondía a esta exigencia, lo que permitía un cálculo relativamente seguro de las mezclas de hormigón. Junto a su capacidad de instrucción, un procedimiento de ensayo debe proporcionar resultados reproducibles. A este respecto, se han llevado a cabo amplios ensayos de comparación dentro del grupo de trabajo del Cembureau, en el grupo de trabajo "RESISTENCIAS" del VDZ y en el Instituto de Investigación de la Industria del Cemento. Las esperanzas respecto a la reproducibilidad, que posiblemente en principio eran un tanto elevadas, no se han cumplido hasta ahora, aun cuando el procedimiento de ensayo en DIN 1164, pág. 7 (1970), se ha descrito muy detalladamente incluyendo las tolerancias permitidas. Sólo en laboratorios que realizan 
un fuerte desembolso en cuanto a climatización, mecánica de la máquina de ensayos, velocidad de carga, etc., $y$ cuyos empleados tienen un trato constante con el ensayo de cementos, puede alcanzarse una desviación standard de 10 a $15 \mathrm{kp} / \mathrm{cm}^{2}$ en pruebas de repetición con el mismo cemento, lo que corresponde a un coeficiente de variación del orden del $2 \%$. En el caso de ensayos comparativos de varios laboratorios con buenas instalaciones, la desviación standard se mantuvo alrededor de $20 \mathrm{kp} / \mathrm{cm}^{2}$. Si se tienen en cuenta los diferentes cementos y todos los laboratorios de ensayo de cemento oficialmente reconocidos, la desviación estandard sobrepasó claramente los $25 \mathrm{kp} / \mathrm{cm}^{2}$. Un resultado idéntico se encontró en un ensayo comparativo entre seis laboratorios de la EWG, en el cual el coeficiente de variación sobrepasó el $5 \%$. Incluso en una ulterior elaboración, con el nuevo procedimiento de revisión debe contarse con que la desviación standard, por término medio, quedará en el orden de magnitud de 20 a $25 \mathrm{kp} / \mathrm{cm}^{2}$. El procedimiento de ensayo empleado hasta la fecha según DIN 1164 antiguo (1942), a pesar del adiestramiento a lo largo de años, tenía una desviación standard algo mayor todavía; por término medio, la que probablemente estaba condicionado, en primer lugar, por la mayor o menor exudación del mortero de ensayo. En conjunto el procedimiento de ensayo DIN 1164 (1970) correspondiente a la ISO, y de acuerdo con el estado actual se puede calificar como el mejor procedimiento en cuanto a la expresividad y reproducibilidad; sin embargo, el procedimiento no es muy sencillo, requiere gran cuidado y tampoco causa pequeños gastos.

(continuará)

\section{B I B L I O G R A. F I A}

[1] Britisch Standard 12: 1958 (Amendments 1960 and 1962) Portland Cement (Ordinary and Rapid-Hardening). British Standards Institution, London.

[2] ASTM C 403-70: Time of setting of concrete mixtures by penetration resistance. 1970 Annual Book of ASTM Standards, Part 10. American Society for Testing and Materials, Philadelphia.

[3] Haegermann, G.: Prüfung der technischen Eigenschaften der Zemente. Handbuch der Werkstoffprüfung, Bd. 3, 2, Aufl. Springer-Verlag Berlin/Göttingen/Heidelberg 1957, S. 356/396.

[4] Cement Standards of the world. Herausgegeben von Cembureau, Paris. Beton-Verlag. Düsseldorf 1968. 